\title{
A Calibrated Measurement of the Near-IR Continuum Sky Brightness Using Magellan/FIRE
}

\author{
Peter W. Sullivan ${ }^{1}$, Robert A. Simcoe ${ }^{1}$
}

\begin{abstract}
We characterize the near-IR sky background from 308 observations with the FIRE spectrograph at Magellan. A subset of 105 observations selected to minimize lunar and thermal effects gives a continuous, median spectrum from 0.83 to $2.5 \mu \mathrm{m}$ which we present in electronic form. The data are used to characterize the broadband continuum emission between atmospheric $\mathrm{OH}$ features and correlate its properties with observing conditions such as lunar angle and time of night. We find that the moon contributes significantly to the inter-line continuum in the $Y$ and $J$ bands whereas the observed $H$ band continuum is dominated by the blended Lorentzian wings of multiple $\mathrm{OH}$ line profiles even at $R=6000$. Lunar effects may be mitigated in $Y$ and $J$ through careful scheduling of observations, but the most ambitious near-IR programs will benefit from allocation during dark observing time if those observations are not limited by read noise. In $Y$ and $J$ our measured continuum exceeds space-based average estimates of the Zodiacal light, but it is not readily identified with known terrestrial foregrounds. If further measurements confirm such a fundamental background it would impact requirements for $\mathrm{OH}$-suppressed instruments operating in this regime.
\end{abstract}

\section{Introduction}

The development of low-noise $\mathrm{HgCdTe}$ focal plane arrays has motivated the construction of a new generation of medium-resolution, near-infrared spectrometers for ground-based telescopes (Simcoe et al. 2008; Herter et al. 2008; Vernet et al. 2011). These instruments resolve the wellknown forest of sky emission line features induced by hydroxyl $(\mathrm{OH})$ ions and other atmospheric molecules. For faint object spectroscopy, instrument sensitivity is therefore limited by the inter-line sky continuum, which is a superposition of terrestrial, astronomical, and instrumental backgrounds.

Reliable estimates of the inter-line IR continuum are critical for the design of successful observations and instruments. Yet calibrated measurements remain sparse in the literature precisely because the strong foreground line emission makes such a calibration very challenging. As we shall demonstrate, the contrast ratio between narrow emission peaks and the broadband continuum approaches 5 magnitudes in the $J$ band and 7 magnitudes in $H$.

\footnotetext{
${ }^{1}$ MIT-Kavli Institute for Astrophysics and Space Research
} 
Attempts to measure the inter-line background accordingly require certain characteristics of instrumentation and data processing. First, the spectral resolution must be sufficiently high to separate lines cleanly. Second, the detector must exhibit low dark current to not overwhelm the sky signal with thermal shot noise. Read noise should also be low, but it may be mitigated by averaging many exposures. Finally, and perhaps most importantly, attention must be given to the reduction of scattered light from optical surfaces in the instrument.

The FIRE spectrograph at Magellan was designed to address these criteria while capturing both line and continuum emission of the sky across the $Y, J, H$, and $K$ bands simultaneously in each exposure. As part of its data processing pipeline, a precise model of the sky from 0.8 to 2.5 $\mu \mathrm{m}$ is generated for each science exposure and saved to disk.

In this paper, we gather these data and present calibrated sky brightness measurements taken from 308 deep exposures obtained across several observing runs since the commissioning of FIRE in March 2010. Section 2 describes the data processing techniques and instrumental considerations used to arrive at the continuum measurement. In Section 3, we examine correlations between the sky brightness and common observational conditions, including lunar phase, moon-object angle, and local time, and we present sky measurements under dark conditions which minimize their influence. Finally, in Section 4, we discuss the implications of our findings for the construction of new instrumentation, planning observations, and allocation of bright versus dark telescope time for near-IR spectroscopic observations.

\section{Methods}

\subsection{Description of FIRE and FIREHOSE}

The Folded-port Infrared Echellette (FIRE) is a single object, cross-dispersed spectrometer which delivers $R=6000$ spectra between 0.82 and 2.5 microns in a single setup (Simcoe et al. 2008). It was commissioned on the Magellan Baade telescope in March 2010 and has been installed permanently since that time.

FIRE uses a substrate-thinned HAWAII-2RG HgCdTe detector and SIDECAR cryogenic readout ASIC to capture the 2-dimensional image of a $6^{\prime \prime}$-long slit over 21 diffraction orders. Characterization of the science array's dark current in a laboratory test environment found a very low count rate of $0.0008 \pm 0.001 \mathrm{e}^{-1} s^{-1}$ per pixel or 2.5 electrons per hour. This is well below the system's read noise and our measured flux from the sky, so dark current does not contribute to the absolute sky value nor its measurement uncertainty.

FIRE operates in quasi-Littrow mode and its cross dispersion is achieved via a network of ZnSe and Infrasil prisms, so the spectral orders are significantly curved and tilted with respect to the detector's pixel basis. We leverage this to obtain sky sampling on sub-integer pixel scales (similar to the drizzle algorithm of Fruchter \& Hook 2002). FIRE's optical design retains several arcseconds 
of dark pixels between orders to facilitate in estimating and removing any diffuse scattered light background. This spacing is largest in $Y$ and $K$ but somewhat smaller in $H$ because of the balance of the prisms' partial dispersions. An additional consequence of the prism cross disperser is variation in plate scale in the spatial direction along the slit from order to order. When estimating surface brightness, we calibrate the pixel scale for each order empirically by measuring its width in pixels and scaling to the fixed $6^{\prime \prime}$ slit length.

FIRE's reduction pipeline (nicknamed FIREHOSE) employs the 2-dimensional sky-subtraction algorithms described by Kelson (2003) combined with optimal extraction (Horne 1986) to produce a 1-dimensional, flux calibrated and telluric-corrected object spectrum. As part of this process, FIREHOSE creates a 2D estimate of the sky using regions of the slit image not illuminated by object flux. Extracting the sky flux from this image using the same spatial profile as the object results in a $1 \mathrm{D}$ sky spectrum. We have archived these sky estimates from several hundred science exposures taken with FIRE since its commissioning. In the raw 2D frames, one can begin to detect continuum emission between the $\mathrm{OH}$ sky lines, but the single-pixel signal-to-noise ratio can fall below unity in the $Y$ and $J$ bands. For estimating the continuum flux, we therefore restricted our

analysis to long science exposures with $t_{\text {exp }} \geq 600$ seconds and good atmospheric conditions. The final set of exposures, taken in lunar conditions ranging from new moon to full moon and pointings through 1.00 to 1.86 airmasses, contains 308 frames that constitute 85 hours of integration time spread over 23 nights on 7 separate observing runs.

\subsection{Electronic Offset Correction}

Although the FIREHOSE pipeline applies gain and offset corrections to the raw 2D images from FIRE, residual offsets remain in the flat-fielded images (Figure 1 1 ). The value of the offset varies from exposure to exposure; furthermore, it differs for each of the four regions on the H2RG detector which feed separate preamplifier channels in the SIDECAR readout ASIC. The resetting of the preamplifiers, which occurs at the beginning of each frame (Loose et al. 2006), is the most likely cause of this offset. Unlike the offset induced by pixel reset, the offset from preamplifier reset is different for each frame and cannot be eliminated through correlated double sampling, Fowler sampling, or sample-up-the-ramp sequences.

During normal data reduction the residual electronic offset is automatically subtracted from object spectra as part of the sky estimate. In order to isolate the sky contribution alone, the electronic offset must be determined independently from the detector pixels lying between each of the echelle orders on the focal plane. From these pixels, we compute a median offset for the four zones corresponding to each SIDECAR preamplifier, shown in Figure 1 b. The typical preamplifier correction is on the order of 5-10 e-, which is comparable to the sky signal level of 5 to $25 e-$ (depending on the grating order) as well as the average read noise for a single pixel, measured from the same non-illuminated pixels to be $7.65 \mathrm{e}-$. However, the large number of pixels used (at least 167,000 per preamplifier channel) and their fairly even distribution across the detector makes the 
uncertainty of this correction much lower than the read noise.

\subsection{Spatially Structured Stray Light Correction}

After the electronic offset is removed for each SIDECAR preamplifier, a residual gradient across the detector remains. Because it resembles a defocused, diffuse version of the echelle illumination, we attribute it to stray light. The chief source of stray light in FIRE is believed to be reflections off the front surface of the detector that subsequently bounce off the facing surface of the last optical element and return to the focal plane. We can again make use of the pixels lying between the echelle orders to interpolate the stray light contribution within the echelle pattern. We fit a third-order, two-dimensional polynomial to the order-masked pixel intensities to construct a stray light map for each frame; an example is shown in Figure 17. As with the electronic offset, the stray light correction of a few electrons is of the order of the sky signal itself at this stage. However, 724,000 pixels are used to generate the stray light estimate, so its uncertainty is negligible relative to that of the sky signal.

We note that the fitted polynomial falls slightly below zero in the corners of the array which might seem unphysical. This arises from the electronic offset correction described in Section 2.2 which over-estimates the offset in the darkest regions of the array. It is not possible to separate the true electronic offset from a uniform diffuse illumination pattern, so we treat the spatially varying portion of the stray light background simply as a higher-order correction to any constant offset. The final, corrected image is shown in Figure 1d.

Although the sky continuum is already visible in the $K$ band in Figure 11d due to thermal emission, the electronic offset and stray light corrections are required to see the sky continuum in $Y, J$, and $H$. Furthermore, the sky signal from our darkest observations fall below the singlepixel read noise floor (for one $600 \mathrm{~s}$ exposure) of $Y_{A B}=18.8, J_{A B}=19.2$, and $H_{A B}=19.5 \mathrm{mag}$ $\operatorname{arcsec}^{-2}$. Averaging over many sky spectra or over several wavelength bins is therefore necessary to reliably measure the sky continuum. Figure 2 shows a stack of 101 echelle images zoomed to the echelle orders corresponding to the $Y$ and $J$ bands and stretched to reveal the sky continuum above the non-illuminated gaps between orders. The stacked images were chosen for their low lunar contribution (further discussed in Section 3.1), so Figure 2 represents a worst-case ratio of sky signal to read noise (approximately 0.3 for a single frame, or 3 for the stack of 101 frames). Because read noise and residual systematics are still much higher than the sky continuum blue-ward of $0.960 \mu \mathrm{m}$, we do not measure the continuum in the $I$ band.

\subsection{Calibration}

Flux calibration of our sky frames is performed by comparison with observations of the hot spectrophotometric standard star GD71 taken in September 2010. The optical efficiency of FIRE 
was determined by comparing extracted counts to a spectrum taken with HST/NICMOS available in the CALSPEC databas 1 . The resulting efficiency of FIRE as a function of wavelength is shown in Figure 3. This single sensitivity function was used to flux calibrate all exposures used in this study.

Flux calibration of FIRE science data is typically achieved using telluric A0V comparison stars rather than white dwarfs, so absolute spectrophotometric standards were only observed for a small number of observing runs. Therefore, we do not have a long term temporal baseline over which to evaluate FIRE's sensitivity from run to run. The GD71 data were taken using a $0.6^{\prime \prime}$ slit and experienced slit losses (which do not apply to the sky surface brightness) since the FWHM of the seeing at the time of the observation was $\sim 0.6^{\prime \prime}$. We performed a crude correction for the slit losses by projecting a circular Gaussian onto the slit profile and estimated the transmitted fraction to be $63 \%$. Given the uncertainty in these parameters, as well as uncertainty in the effective area and reflectivity of the Magellan telescope mirrors, we conservatively estimate our systematic uncertainty in absolute calibration of the sky flux at $\sim 15 \%$.

\subsection{FIRE's Line Spread Function}

It has been noted that a significant contribution to the inter-line background may arise from broad wings of the Line Spread Function (LSF) which spread light out of the $\mathrm{OH}$ lines. Such wings might originate from scattering at the grating or other optical surfaces in IR instruments (Bland-Hawthorn et al. 2004). If substantial, this effect would reduce the number of pixels that achieve true background levels. The contribution of the LSF wings to the apparent sky continuum is most important in the $H$ and $K$ bands, where the $\mathrm{OH}$ lines are stronger than in the $Y$ and $J$ by 1-2 orders of magnitude.

In Figure 4, we show a high signal-to-noise ratio composite arc line profile from FIRE where the $x$ axis is scaled to units of constant velocity $\Delta v / c$ or $\Delta \lambda / \lambda$. The data were derived from a deep stack of $20 \mathrm{ThAr}$ arc lamp exposures read at Fowler-4 to bring out the weak wings of the profile. The core of the profile is taken from a bright but unsaturated spectral line while the wing profile is taken from a nearby line which is saturated in the core. The relative normalizations of the core and wings were set by matching the profile in overlapping regions. The black dots represent individual 2D pixel estimates of the flux; several gaps have been masked to eliminate nearby faint ThAr lines (a handful at $\Delta v / c<-0.005$ were left in the Figure for reference).

Using these data we explored empirical fits to the LSF functional form. First, we verified that the core of the LSF matched expectations from FIRE's design specifications. This test is illustrated with the red curve, which shows a 4-pixel-wide tophat function (the transmission of a $0.6^{\prime \prime}$ slit) convolved with a Gaussian with a FWHM of 2.0 pixels (FIRE's optical specification), and

\footnotetext{
${ }^{1}$ http://www.stsci.edu/hst/observatory/cdbs/calspec.html
} 
further convolved with a kernel describing the MTF degradation from inter-pixel capacitance in the H2RG detector. The latter kernel taken from measurements of the inter-pixel capacitance of FIRE's detector made in a controlled environment, which we model as a leakage of $1.6 \%$ between adjacent pixels. This core model (Figure 4 inset) is an excellent match to the measured LSF out to $\sim 3 \sigma$; the wings of the profile only begin to contribute when the core has fallen to $0.1-0.2 \%$ of its central value. For reference, the diffraction pattern expected from projecting a uniform circular beam onto FIRE's grating is shown in blue; since the spectral resolution is limited by the slit, it does not dominate the LSF.

At larger separations the wings are much more prominent than the Gaussian core would indicate; these most likely originate from scattering off of the gratings and spectrograph optics. Woods et al. (1994) studied this effect in detail, fitting the LSF with a combined Gaussian + Lorentzian + constant offset. We performed a similar fit, shown as the green line. However, FIRE's LSF appears to have wings on two different spatial scales such that a combination of two Lorenzian profiles were required: one to fit the shoulder near $|\Delta \lambda / \lambda|<0.002$ and another broad component to model power scattered to larger wavelength separations. In the later discussion we use this model as our baseline empirical LSF.

\subsection{The Continuum Flux Measurement}

The inter-line sky continuum was computed for each observation separately for the $Y, J$, and $H$ bands by finding the mode of the sky flux through an empirical kernel density estimate. The continuum flux levels give a strong peak in the probability density of the flux (and a well-defined mode), but the sky emission lines are rejected since they can take on virtually any amplitude above the continuum level. Since thousands of spectral bins contribute to the continuum measurement, the low signal-to-noise ratio for a single pixel is easily improved by this approach. Only in the $K$

band is the continuum level ill-defined because thermal emission from the telescope and atmosphere causes the background to rise sharply with wavelength. Since these effects are site-specific and do not reflect the fundamental sky background, we do not compute the $K$ sky brightness.

\section{Data and Discussion}

\subsection{Effects of Moon, Observation Time, OH Flux}

Since the sky continuum has been quantified under a variety of observing conditions, it is possible to determine how the dark sky levels quantified in Section 2.6 change with the influence of moon, local time, and the $\mathrm{OH}$ line flux. It is well-established that lunar illumination increases the inter-line sky continuum in the optical; the short-wave end of FIRE overlaps with this region and may suffer from lunar sky brightening as well. As shown in Figure 5, the sky continuum levels 
measured in the $Y$ band do indeed decrease with angular separation from the moon and increase with moon elevation. The horizontal portions of the fits show the mean sky level under moonless conditions, and the sloping portions show a linear least-squares fit to the sky magnitude from the observations where the moon is present. The slopes computed in all three bands are summarized Table1. The sky continuum is most strongly correlated with moon in the $Y$ and uncorrelated in $H$. Due to the desire to observe at low airmass, observations taken with a high lunar elevation largely coincide with observations having a small moon-object angle. This degeneracy makes it difficult to determine which factor has a greater impact upon the sky continuum in the $Y$ and $J$.

At longer wavelengths, thermal emission from the telescope, instrument, and atmosphere decreases as the night progresses. In addition, the $\mathrm{OH}$ line strength is strongest in the evening twilight and can induce a higher measured continuum via the wings of the LSF. Thermal and $\mathrm{OH}$ flux dependencies therefore trend with the local time, which indeed is moderately correlated with sky continuum in the $H$ band. In Figure 6, a linear fit to local time has been performed on the observations having a moon angle of more than 90 degrees and presented in Table 1. The $\mathrm{OH}$ line flux can be separately measured as the integrated flux in the band above twice the continuum level. The correlation between the $H$ continuum and $\mathrm{OH}$ flux, as shown in Table 1, is quite strong, suggesting that the Lorentzian wings of the LSF may account for the observed inter-line continuum in $H$. Due to the noticeable effects of thermal and $\mathrm{OH}$ emission, we do not find observations taken before 2200 local standard time to be "dark".

As summarized in Table1, the $Y$ band continuum shows no significant dependence on the local time or $\mathrm{OH}$ flux, and the $H$ band continuum shows weak dependence on lunar effects. Furthermore, the sky continuum shows no clear correlations with ecliptic declination, airmass, or solar angle in any of the bands.

\subsection{Dark Sky Measurements}

The distribution of measured continuum values for each band are shown in Figure 7 . To quantify the true sky continuum level under dark conditions, we selected the sky measurements with minimal contamination from the moon and thermal emission. As justified in the previous section, we define "dark" observations to be those taken with a moon-object angle of at least 90 degrees and no earlier than two hours before local midnight. There were 105 observations which met these criteria (constituting 31 hours of integration time), and the distributions of the dark subsets are also shown in Figure 7.

For the dark observations, we measure mean inter-line sky continuum levels of $Y_{A B}=20.05$ $\pm 0.04, J_{A B}=19.55 \pm 0.03$, and $H_{A B}=18.80 \pm 0.02 \mathrm{mag} \operatorname{arcsec}^{-2}$. Besides the statistical uncertainties listed here, each measurement has a systematic uncertainty of $+0.15 /-0.18 \mathrm{AB}$ mag $\operatorname{arcsec}^{-2}$ that stems from the efficiency calculation described above. We also computed the median sky spectrum that is available in electronic form. The sky spectrum has read noise floors of $Y_{A B}=21.35$, 
$J_{A B}=21.75$, and $H_{A B}=22.07$, which is below the continuum levels by more than one magnitude for wavelengths greater than $0.960 \mu \mathrm{m}$.

\subsection{Comparison with Previous Sky Estimates}

When developing designs for FIRE, we relied on two estimates of the sky continuum: Maihara et al. (1993) and the Gemini Observatory sky mode2. The Gemini model was generated using a combination of ATRAN (Lord 1992) models and the theoretical OH emission spectrum of Rousselot et al. (2000). Since FIRE's construction, the Gemini model has been revised, so we compare to the latest version (uploaded 16 April 2010). We also compare to the measurements of Cuby et al. (2000) and Ellis et al. (2012).

Maihara's measurement, made with the UH 2.2 meter telescope on Mauna Kea, reports a flux of $590( \pm 140) \gamma^{-1} \mathrm{~s}^{-2} \mu \mathrm{m}^{-1} \operatorname{arcsec}^{-2}$ or $19.4( \pm 0.3) \mathrm{AB}$ mag $\operatorname{arcsec}^{-2}$ over the range of 1.661 to $1.669 \mu \mathrm{m}$. A direct comparison to our measurement is complicated by low-level emission features near $1.665 \mu \mathrm{m}$ (attributed to atmospheric methane) that are not apparent in the Mauna Kea data. Considering only the range of 1.662 to $1.663 \mu \mathrm{m}$ (as shown in Figure 8) gives a sky flux of 670 $( \pm 200) \gamma \mathrm{s}^{-1} \mathrm{~m}^{-2} \mu \mathrm{m}^{-1} \operatorname{arcsec}^{-2}$ or $19.2( \pm 0.2) \mathrm{AB}$ mag $\operatorname{arcsec}^{-2}$ measured by FIRE, which is well within the upper bound of Maihara's uncertainty. Our measurement across the entire $H$ band of $18.8( \pm 0.2) \mathrm{AB}$ mag $\operatorname{arcsec}^{-2}$ is also consistent with Ellis et al. (2012), who measures $860( \pm 210)$ $\gamma \mathrm{s}^{-1} \mathrm{~m}^{-2} \mu \mathrm{m}^{-1} \operatorname{arcsec}^{-2}$ or $19.0( \pm 0.3) \mathrm{AB}$ mag $\operatorname{arcsec}^{-2}$ in the $H$ band.

The Gemini sky model shows a darker continuum flux of $19.63 \mathrm{AB}$ mag $\operatorname{arcsec}^{-2}$ at $1.663 \mu \mathrm{m}$. However, at $1.25 \mu \mathrm{m}$, the Gemini model gives 19.35 versus our measurement of $19.55 \mathrm{AB}$ mag $\operatorname{arcsec}^{-2}$ across $J$, and at $1.02 \mu \mathrm{m}$, the model gives 19.23 versus our measurement of $20.05 \mathrm{AB}$ mag $\operatorname{arcsec}^{-2}$ across $Y$. Maihara's measurements do not cover the $J$ or $Y$ bands, but provisional measurements of the inter-line continuum in $H$ and $J$ were made during commissioning of the ISAAC spectrometer on the ESO/VLT (Cuby et al. 2000). The ISAAC team reports backgrounds of 17.88 in $H$ and $18.94 \mathrm{AB}$ mag $\operatorname{arcsec}^{-2}$ in $J$; in linear units this is 2.3 times higher than our measurement in $H$ and $\sim 75 \%$ higher than our measurement in $J$. One might expect a significantly higher background at the lower and warmer site of the Magellan Telescopes at Las Campanas relative to Mauna Kea or Paranal, but we find this is not the case.

\subsection{What Fraction of the Sky is OH-Free?}

For the design of IR spectrometers, it is vital to understand what fraction of the infrared sky is "clean" of $\mathrm{OH}$ contamination and how this quantity varies with spectral resolution. This is

\footnotetext{
${ }^{2}$ http://www.gemini.edu/?q=node/10787
} 
particularly true in light of the broad wings present in the LSF for FIRE (or indeed any known spectrometer). The above analysis indicates that some $\mathrm{OH}$ flux enters the continuum (especially in the $H$ ), but it is possible that all of the broadband flux we have attributed to the continuum is in fact a superposition of wings from many neighboring OH features (Ellis \& Bland-Hawthorn 2008).

With a working model of the LSF, we may test this hypothesis by constructing a simulated, high-resolution sky containing only $\mathrm{OH}$ lines, convolving it with the measured LSF and comparing its resultant inter-line continuum with our measured values. To this end, we used the $\mathrm{OH}$ line lists of Rousselot et al. (2000) to set the wavelengths and relative intensities of the $\mathrm{OH}$ forest lines in a high resolution $(R=30,000)$ spectrum consisting of simple Gaussian profiles. All line peak heights were normalized to the data using a single, constant scale factor, which was determined by enforcing that the average total flux in the model lines equal the average total flux in our line measurements for the true sky. For the high resolution spectrum this corresponds to narrower and taller peaks but with the same integrated area. We then convolve with the LSF determined above, finding (as expected) that the height and width of the individual lines match in detail.

Figure 9 illustrates the result of this calculation, where the red curve shows the model $\mathrm{OH}$ forest convolved with the LSF and the black shows our measured median sky spectrum. We find that in $H$, scattered light from the wings of the LSF can indeed account for the full measured background flux seen in FIRE, so the two curves agree quite well. However, in $Y$ and $J$ the convolved curves fall well below our measured background, indicating that the continuum in these bands arises from a source other than the $\mathrm{OH}$ forest. The read noise floor of our median sky spectrum and HST zodiacal light levels are also plotted, but they fall far below our measured spectrum.

This dichotomy may be easily understood in terms of dynamic range and line density. The LSF presented in Figure 4 clearly shows that for intrinsic line-to-continuum ratios smaller than five magnitudes, the inter-line spectral regions never "see" the LSF wings, so only the core emerges from the background. At line-to-continuum ratios $\gtrsim 5 \mathrm{mag}$, the wings become more prominent and can extend for many pixels. In $Y$ and $J$, our observed line-to-continuum ratio of 2-5 mag puts the blended $\mathrm{OH}$ background well below the continuum value. Only in $H$ are we in the region where $\mathrm{OH}$ blending dominates.

To address the question heading this section, it appears that there is no truly OH-free spectrum in FIRE's $H$ band channel as blending of the LSF's wings limits the background, commensurate with the findings of Ellis \& Bland-Hawthorn (2008). Our extrapolation to zero OH flux (Table 1) suggests that a continuum of $H_{A B}=19.4 \mathrm{AB}$ mag $\operatorname{arcsec}^{-2}$ may be achievable. Blue-ward of $H$, a fundamental background is reached by significant proportions of the spectrum. To better quantify this statement, we plot in Figure 10 the cumulative sky flux distribution renormalized so that a value of unity corresponds to the continuum level over each bandpass. In $Y$, approximately $30 \%$ of the spectrum is at the background level and $75 \%$ of the spectrum is within a factor of 6 of the background. In $J$ and $H$, less than $25 \%$ is at the background, and the $75 \%$ mark is only reached at 10 times the background. 
To quantify the effects of spectral resolution on the $Y$ and $J$ measurements, we recalculated the cumulative sky flux distribution for degraded instrument resolutions. FIRE's resolution is slitlimited so one may convolve with tophat kernels of varying width to simulate the effect of changing slits. However, in this analysis we wished to simulate instruments of varying spectral resolution that are assumed to critically sample a LSF at a specified resolution, so a Gaussian kernel is more appropriate. There is no need to include the Lorentzian wings in the convolution since the continuum level dominates in these bands.

Following Martini \& DePov (2000), we plot in Figure 11 the proportion of spectral bins which are read-noise dominated versus resolution. We see that as $R$ increases from 500 to 3000, a critical change occurs as continuum begins to emerge between $\mathrm{OH}$ peaks. Martini assumed a lower value of the detector read noise (5 e- versus our operational value of 7.65 e-), so we compensate with a longer effective exposure time $(1500 \mathrm{~s}$ versus $600 \mathrm{~s})$ in order to make the plots comparable. In the $J$ band, we arrive at essentially the same proportion of read noise-dominated bins at $\mathrm{R}=6000$ as Martini, but at $\mathrm{R}=500$, we predict that $33 \%$ of spectral bins are read noise-dominated where Martini predicts $0 \%$. In the $Y$ band, we show that at $\mathrm{R}=6000,83 \%$ of spectral bins are read noise-dominated, and at $\mathrm{R}=5000,64 \%$.

Since the spectral bins at $R=6000$ that reach the continuum level are read noise-dominated, one can alternatively determine the exposure time required to reach sky-dominated noise. This is a critical limit to calculate for any instrument observing faint objects. Assuming a sky background of $20.05 \mathrm{AB}$ mag $\operatorname{arcsec}^{-2}$ in $Y$, FIRE receives a photon count from the sky of

$$
f=0.051 \gamma \sec ^{-1}\left(\frac{D_{t e l}}{6.5 \mathrm{~m}}\right)^{2}\left(\frac{\Delta v_{\text {pix }}}{12.5 \mathrm{~km} / \mathrm{s}}\right)^{-1}\left(\frac{w_{\text {slit }}}{0.6^{\prime \prime}}\right)\left(\frac{h_{\text {pix }}}{0.125^{\prime \prime}}\right)
$$

where we have used scalings for the telescope aperture $D_{t e l}$, the velocity interval per pixel $\Delta v_{p i x}$, the slit width $w_{\text {slit }}$ and pixel scale $h_{\text {pix }}$ in the spatial direction appropriate for Magellan/FIRE. To translate to a noise budget, we assume $\sim 24 \%$ instrument efficiency in $Y$ and $J$ (Figure 3) and a read noise RMS of $7.65 e^{-}$, appropriate for Fowler-8 or long Sample-Up-The-Ramp integrations. For these typical conditions, detector read noise dominates the total budget for exposures shorter than $t_{\text {exp }} \approx 4800$ seconds, or 80 minutes. Our original designs for FIRE were based on a slightly brighter estimate of the inter-line continuum, which would have delivered sky-dominated exposures

in $\lesssim 10$ minutes. Hence, this minimum time to sky-dominated noise is longer than we consider optimal.

\subsection{Sources of Inter-Line Continuum Emission in the $Y$ and $J$ bands}

The inter-line continuum in $Y$ and $J$ substantially exceeds our scattered light models derived from the empirical LSF by 1-2 magnitudes or a factor of 2 to 6 . This suggests the existence of another fundamental diffuse background for terrestrial observations, which would have implications for the efficacy of dark and/or OH-suppressed imaging and spectroscopy in this regime. However, 
this detection remains puzzling as there is not a readily identifiable emission mechanism in the Earth's atmosphere that would give rise to such a background.

We were initially concerned that our "measurement" might actually reflect a read noise floor rather than a statistically significant detection. However, Figure 2 illustrates an enhancement in the diffuse flux between adjacent $\mathrm{OH}$ lines relative to the non-illuminated gaps between echelle orders even under dark conditions. Furthermore, in Figure 9, the effective surface brightness of the read noise (defined as the magnitude corresponding to a $1 \sigma$ constant offset from zero flux) is plotted with the sky measurement. Comparison of the read noise floor and the measurement curve clearly shows detection at a signal-to-noise ratio varying with wavelength from $\sim 2.3 \sigma$ to $\sim 6 \sigma$.

Beyond instrumental effects, reflected Zodiacal light (ZL) is considered the fundamental continuum limit in the near-IR once the $\mathrm{OH}$ forest has been mitigated. This background has been measured at $1.2 \mu \mathrm{m}$ from orbit using HST/NICMOS with an average value of $22.4 \mathrm{AB}$ mag $\operatorname{arcsec}^{-2}$ (Stiavelli 2001). This is 2.8 magnitudes (or a factor of $\sim 14$ ) fainter than our measurement at $J$.

In addition to instrument-scattered $\mathrm{OH}$, read noise, and the Zodical backgrounds, other possible sources of diffuse light include unresolved stellar populations (primarily Red Giants in the near-IR) as well as tropospheric scattering of all the above backgrounds combined with terrestrial light pollution (Leinert et al. 1998). We expect the contribution from unresolved stars with $J>20$ to be minimized since most of the data used to determine the stack were obtained from observations of high-redshift quasars, which are located preferentially out of the Galactic plane. At high galactic latitude $b$, the integrated $1.25 \mu \mathrm{m}$ stellar background estimated from DIRBE is 10-100 times lower than in the plane and should in fact fall somewhat below the average ZL background (Leinert et al. 1998). Even the resolved stellar background will have a small fraction of radiation scattered by the troposphere into a diffuse component, but it too is mitigated at high $b$. Atmospheric turbulence and scattering of the very strong $\mathrm{OH}$ forest does not distort the spectrum (at the resolutions considered here) and should not spread on-resonance $\mathrm{OH}$ emission into the dark inter-line regions.

Light pollution is also expected to play a minimal role as Las Campanas is a substantially dark site and the stacks presented here were selected for dark lunar conditions. Presently, measurements do not exist for the level of light pollution at LCO, but corresponding studies at CTIO/Cerro Pachon 3 - both of which are closer to the cities of La Serena/Coquimbo-remain quite dark and the contribution of light pollution falls below that of the ZL over most of the sky.

It appears that a robust measurement of the $Y$ and $J$ backgrounds will remain challenging for some time to come, and further estimates of this background with other instruments of varying resolution will be important for verifying the results reported here. Despite our careful attempts to correct for diffuse and grating-scattered light in our optical system, there is a faint signal detected which cannot be identified with a simple atmospheric emission mechanism. Since this emission is only detected at $\sim 2-5 \sigma$ significance in a $>20$ hour stacked spectrum on a 6.5 meter telescope,

\footnotetext{
${ }^{3}$ http://www.ctio.noao.edu/site/pachonsky/
} 
we may be susceptible to systematic effects beyond those considered here. Since we are read noisedominated in this regime for single exposures, it will be particularly important to test this result against spectra obtained at lower resolution in low-background instruments with HAWAII-2RG detectors such as the newly-commissioned MOSFIRE spectrograph (McLean et al. 2010). These may be more sensitive to blended wings of the $\mathrm{OH}$ lines but could provide a crucial check of the broadband signal in relatively un-blended regions.

\section{Implications for Telescope Allocation and Instrument Design}

Traditionally, infrared spectrometers (and imagers) are scheduled for use during the bright phase of the lunar cycle under the premise that the $\mathrm{OH}$ sky is of comparable or greater brightness than the moon and therefore dominates the background. This is clearly the case for imagers and low-resolution spectrometers, but with low-background, moderate-resolution instruments, we must consider whether there is a regime where the moon makes a substantial contribution to the noise background. Such a finding would have implications for best observing practices and affect whether telescope allocation committees should consider proposals to perform IR spectroscopy during coveted dark time.

Our simple regression and $\mathrm{OH}$ blending analyses indicate that lunar illumination has a negligible impact in the $H$ and $K$ bands but does matter in $Y$ and $J$. Our data show that when the moon is set and/or dark, the background converges to its lowest values, between $Y_{A B}=19.5$ and 20.5. When the moon is nearby or overhead, the background is over a magnitude $(2.5 \times)$ brighter. This amounts to a $58 \%$ increase in noise over the dark-sky case, if the instrument is backgroundlimited. Put differently, the same $Y$-band observation will achieve, on average, a $58 \%$ improvement in signal-to-noise ratio when undertaken in dark skies, which is equivalent to increasing the telescope's primary aperture by the same factor.

We therefore conclude that the sky is sufficiently dark in $Y$ and $J$ that the most aggressive science programs, which are sky-noise dominated, would indeed benefit from scheduling during gray or dark time. Some portion of the benefits may be obtained by careful scheduling of targets

to maximize moon angle, ideally to $\gtrsim 60$ degrees. During the full moon this becomes impractical when coupled with the desire to observe at low airmass, so some compromise must be reached. Science programs relying primarily on $H$ or $K$ may be observed equally well during bright or dark time.

The low sky background and associated tradeoff with read noise have informed our observing and data reduction strategies for FIRE (particularly for high-redshift targets.) Since the exposure times required to achieve sky dominated noise are significantly longer than the coherence time of the $\mathrm{OH}$ sky lines, standard pairwise image subtraction techniques (e.g. "ABBA" nods) do not perform well for sky subtraction. Instead, we now acquire the faintest targets in long sample-upthe-ramp integrations ( $~ 30$ minutes) and reduce each frame using a sky model generated on the fly. 
This approach is time consuming and computationally expensive, but it has the dual advantages of mitigating read noise and increasing the signal-to-noise ratio relative to pairwise subtraction by a factor of $\sim \sqrt{2}$.

Our sky measurements may warrant consideration in the design of future IR spectrometers for large aperture telescopes. As one moves to high spectral resolution or narrow-slit, AO-fed instrumentation, the benefits for faint object work may not be fully realized unless telescope aperture keeps pace with the associated reduction in sky background or if there is a concerted effort to battle sensor read noise to even lower values. Both approaches require considerable resources and careful trade studies would be needed to optimize allocation between the two.

If the inter-line background measured in the $Y$ and $J$ is supported by further observations, then it would imply that ground-based observations could only reach within $\sim 3$ magnitudes of the Zodiacal background in the near-IR, or slightly more than a factor of 10 . This background would slightly weaken the case for OH-suppressed instruments (e.g., Ellis et al. 2012), but it would also lessen the technical requirements for $\mathrm{OH}$-suppressed instruments to achieve background-limited performance in $J$ from $\sim 30-35 \mathrm{~dB}$ narrowband attenuation to $\sim 20 \mathrm{~dB}$. These arguments are particularly germane for studies of the Lyman alpha transition in high-redshift objects since it is observable in the dark portions of $Y$ and $J$ bands throughout the epoch of cosmic reionization.

\section{Conclusions}

We have presented a composite spectrum of the near-infrared night sky obtained by stacking 308 exposures obtained with Magellan/FIRE on 23 nights over seven observing runs. Careful attention was given to correcting electronic artifacts from the detector and scattered light in the instrument. Absolute flux calibration was taken from hot spectrophotometric standards, although the systematic error in the instrument efficiency is uncertain at the $\sim 15 \%$ level because of slit losses. Analysis of a high signal-to-noise arc lamp composite indicates a small amount of $\mathrm{OH}$ line flux is scattered into broad Lorentzian wings at the level of $\sim 10^{-4}$ times the peak line intensity. We explored measurements of FIRE's inter-line sky continuum and compared to previous estimates with the following main results:

1. The mean of the inter-line continuum falls at $Y_{A B}=20.05 \pm 0.04, J_{A B}=19.55 \pm 0.03$, and $H_{A B}=18.80 \pm 0.02$ (stat.) \pm 0.2 (sys.) mag $\operatorname{arcsec}^{-2}$. This is consistent with what is reported in Maihara et al. (1993) for the $H$ band.

2. The $H$ band background correlates most strongly with $\mathrm{OH}$ intensity since the $H$ band "continuum" is largely a superposition of scattered light from $\mathrm{OH}$ emission features. OH intensity decreases after sunset, giving a moderate correlation between $H$ continuum flux and time of night, but thermal emission also contributes. Our $H$ band measurement and that of Maihara et al. (1993) may not achieve the true $H$ continuum, which we extrapolate to being 
19.4 AB mag $\operatorname{arcsec}^{-2}$.

3. The $Y$ and $J$ band inter-line continuum fluxes correlate with moon-object angle and moon elevation, which are degenerate in our data set. Observations obtained at $\lesssim 30$ degrees from

a gibbous to full moon exhibit backgrounds 2.5 times brighter on average than those from a dark sky. This trend is much weaker in $H$ and not seen in $K$.

4. Under dark conditions, the $Y$ and $J$ continua are still higher than the predicted signal from scattered $\mathrm{OH}$ emission, and our measurements seem to detect a true broadband background. At $R \sim 6000,60-80 \%$ of spectral bins achieve this background level. If real, it exceeds the average level of Zodiacal light measured by HST/NICMOS, yet it is not easily explained by known terrestrial foregrounds.

5. The most challenging programs in $Y$ and $J$ will benefit from obtaining telescope allocations during the dark half of the lunar cycle provided that instruments are sufficiently sensitive (or exposures are sufficiently long) for sky noise to dominate the higher read noise of CMOS/HgCdTe detectors over CCDs.

The authors wish to thank the staff of the Magellan Telescopes and Las Campanas Observatory for their assistance in obtaining the observations described herein. The majority of the data were taken for programs supported under NSF Grants AST-0908920 and AST-1109115. RAS thanks D. Schelegel, S. Perlmutter, and D. Fabricant for providing continued motivation to see this analysis through. RAS was supported during this research by the Adam J. Burgasser Chair in Astrophysics.

\section{REFERENCES}

Bland-Hawthorn, J., Englund, M., \& Edvell, G. 2004, Optics Express, 12, 5902

Cuby, J. G., Lidman, C., \& Moutou, C. 2000, ESO Messenger, 101, 2

Ellis, S. C., \& Bland-Hawthorn, J. 2008, MNRAS, 386, 47

Ellis, S. C., et al. 2012, MNRAS, 425, 1682

Fruchter, A. S., \& Hook, R. N. 2002, PASP, 114, 144

Herter, T. L., et al. 2008, in Society of Photo-Optical Instrumentation Engineers (SPIE) Conference Series, Vol. 7014, Society of Photo-Optical Instrumentation Engineers (SPIE) Conference Series

Horne, K. 1986, PASP, 98, 609

Kelson, D. D. 2003, PASP, 115, 688 
Leinert, C., et al. 1998, A\&AS, 127, 1

Loose, M., Beletic, J., Garnett, J., \& Muradian, N. 2006, in Society of Photo-Optical Instrumentation Engineers (SPIE) Conference Series, Vol. 6265

Lord, S. D. 1992, in NASA Technical Memorandum, Vol. 103957

Maihara, T., Iwamuro, F., Yamashita, T., Hall, D. N. B., Cowie, L. L., Tokunaga, A. T., \& Pickles, A. 1993, PASP, 105, 940

Martini, P., \& DePoy, D. L. 2000, in Society of Photo-Optical Instrumentation Engineers (SPIE) Conference Series, Vol. 4008, Society of Photo-Optical Instrumentation Engineers (SPIE) Conference Series, ed. M. Iye \& A. F. Moorwood, 695-702

McLean, I. S., et al. 2010, in Society of Photo-Optical Instrumentation Engineers (SPIE) Conference Series, Vol. 7735, Society of Photo-Optical Instrumentation Engineers (SPIE) Conference Series

Rousselot, P., Lidman, C., Cuby, J.-G., Moreels, G., \& Monnet, G. 2000, A\&A, 354, 1134

Simcoe, R. A., et al. 2008, in Society of Photo-Optical Instrumentation Engineers (SPIE) Conference Series, Vol. 7014

Stiavelli, M. 2001, in WFC3 ISR-2002-02

Vernet, J., et al. 2011, A\&A, 536, A105

Woods, T. N., Wrigley, R. N., Rottman, G. J., \& Haring, R. E. 1994, Applied Optics, 33, 4273 
Table 1: Correlations between sky continuum and lunar, thermal, and hydroxyl effects.

\begin{tabular}{|c|c|c|c|c|c|c|}
\hline & \multicolumn{3}{|c|}{ Fit to Moon Angle } & \multicolumn{3}{|c|}{ Fit to Moon Elevation } \\
\hline & Value $^{\mathrm{a}}$ at $90^{\circ}$ & Slope ${ }^{\mathrm{b}}$ & $R^{2}$ & Value $^{\mathrm{a}}$ at $0^{\circ}$ & Slope ${ }^{b}$ & $R^{2}$ \\
\hline $\bar{Y}$ & 20.01 & 17.9 & 0.273 & 19.99 & -19.5 & 0.194 \\
\hline$J$ & 19.50 & 11.2 & 0.245 & 19.47 & -10.7 & 0.110 \\
\hline \multirow[t]{3}{*}{$H$} & 18.71 & 4.11 & 0.093 & 18.68 & -2.50 & 0.010 \\
\hline & \multicolumn{3}{|c|}{ Fit to Local Time } & \multicolumn{3}{|c|}{ Fit to $\mathrm{OH}$ Flux } \\
\hline & Value $^{\mathrm{a}}$ at Midnight & Slope & $R^{2}$ & Value $^{\mathrm{d}}$ at 0 Flux & Slope $e^{e}$ & $R^{2}$ \\
\hline$Y$ & 20.05 & 56.0 & 0.119 & 0.0312 & 0.013 & 0.025 \\
\hline$J$ & 19.50 & 54.3 & 0.237 & 0.0543 & 0.003 & 0.044 \\
\hline$H$ & 18.69 & 62.7 & 0.461 & 0.0619 & 0.022 & 0.462 \\
\hline
\end{tabular}

${ }^{a} \mathrm{AB}$ mag $\operatorname{arcsec}^{-2}$.

${ }^{b} \mathrm{AB}$ mmag $\operatorname{arcsec}^{-2} \mathrm{deg}^{-1}$.

${ }^{c} \mathrm{AB}$ mmag $\operatorname{arcsec}^{-2} \mathrm{hr}^{-1}$.

${ }^{d} \mathrm{mJy} \operatorname{arcsec}^{-2}$.

${ }^{e}$ Dimensionless in linear units, e.g. mJy $\operatorname{arcsec}^{-2}$ per mJy $\operatorname{arcsec}^{-2}$. 


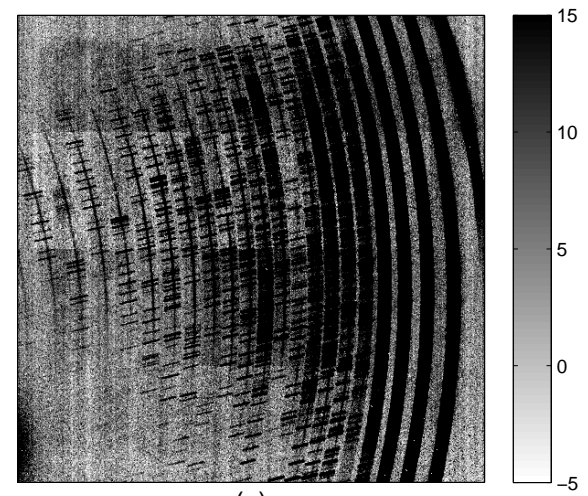

(a)

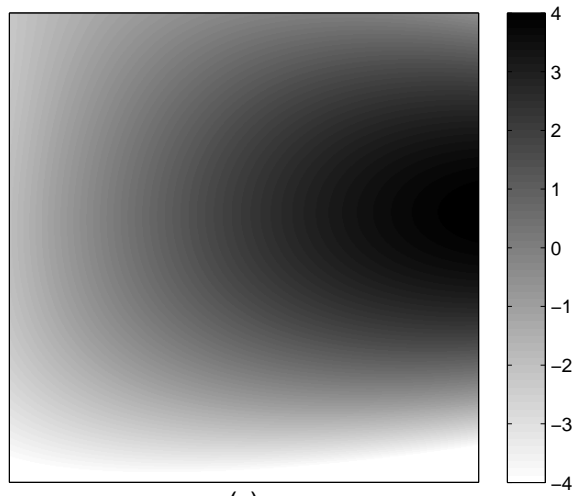

(c)

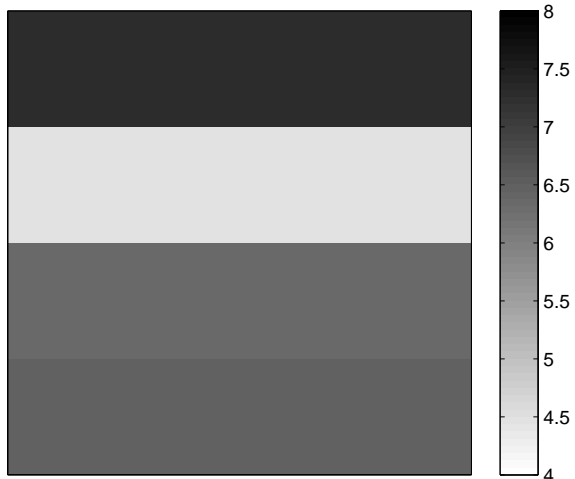

(b)

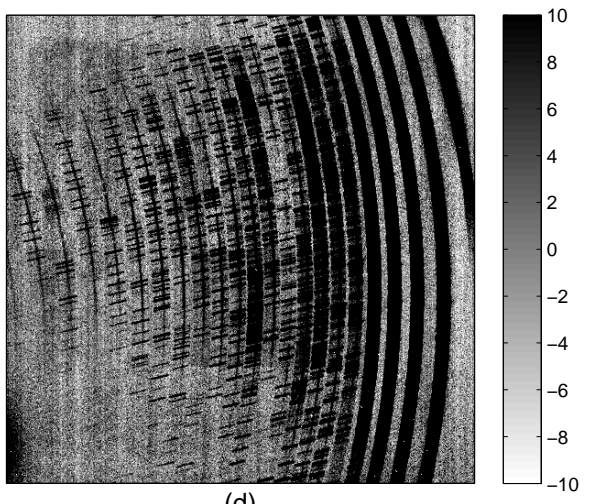

(d)

Fig. 1.- A typical flat-fielded image is shown in (a). From the non-illuminated gaps between grating orders, we can estimate the offset for each SIDECAR preamplifier (b) and the stray-light contribution (c). Subtracting both from the flat-fielded image produces the final result in (d). In practice, the offset and stray light subtraction steps are performed on the 1D sky spectrum estimates produced for each echelle order. Note that all four images are in units of photoelectrons but stretched differently. 


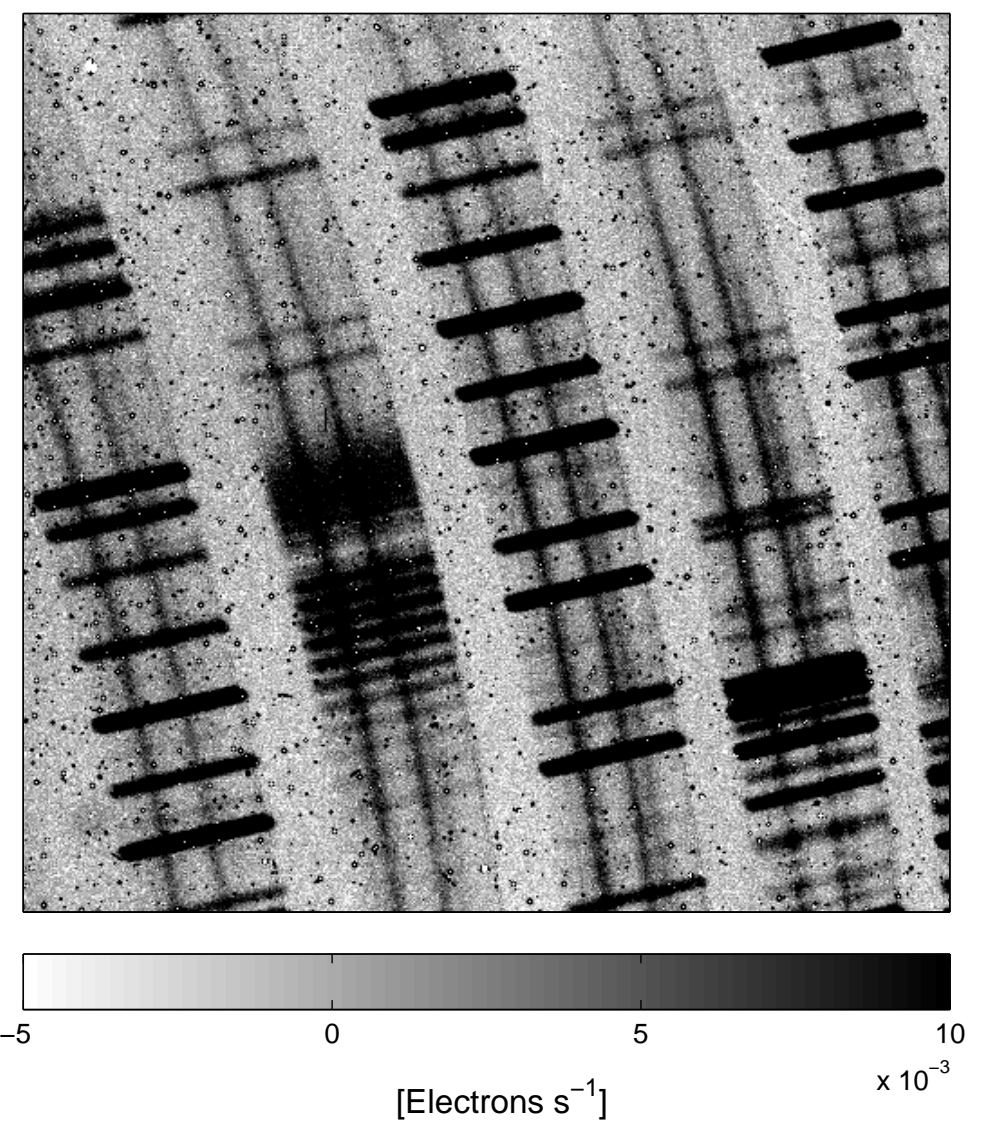

Fig. 2.- Once the subtractions shown in Figure 1 are applied, a stack of 101 images begins to reveal a faint sky continuum in between sky emission lines across the slit. Here, the orders of the echelle image corresponding to the $Y$ and $J$ bands are shown in units of photoelectrons per second. The ratio of sky signal to read noise is approximately 3. The A and B object positions are one- and two-thirds across the slit, where a superposition of object flux is visible perpendicular to the sky lines. The 1D sky spectrum is interpolated at the spatial position of the object flux. Nonuniformities in the slit illumination profile are corrected at a later step in the pipeline, so a slight brightening towards the edge of the slit is visible. The images chosen for the stacking were taken with the moon below the horizon for minimum lunar contribution to the continuum . 


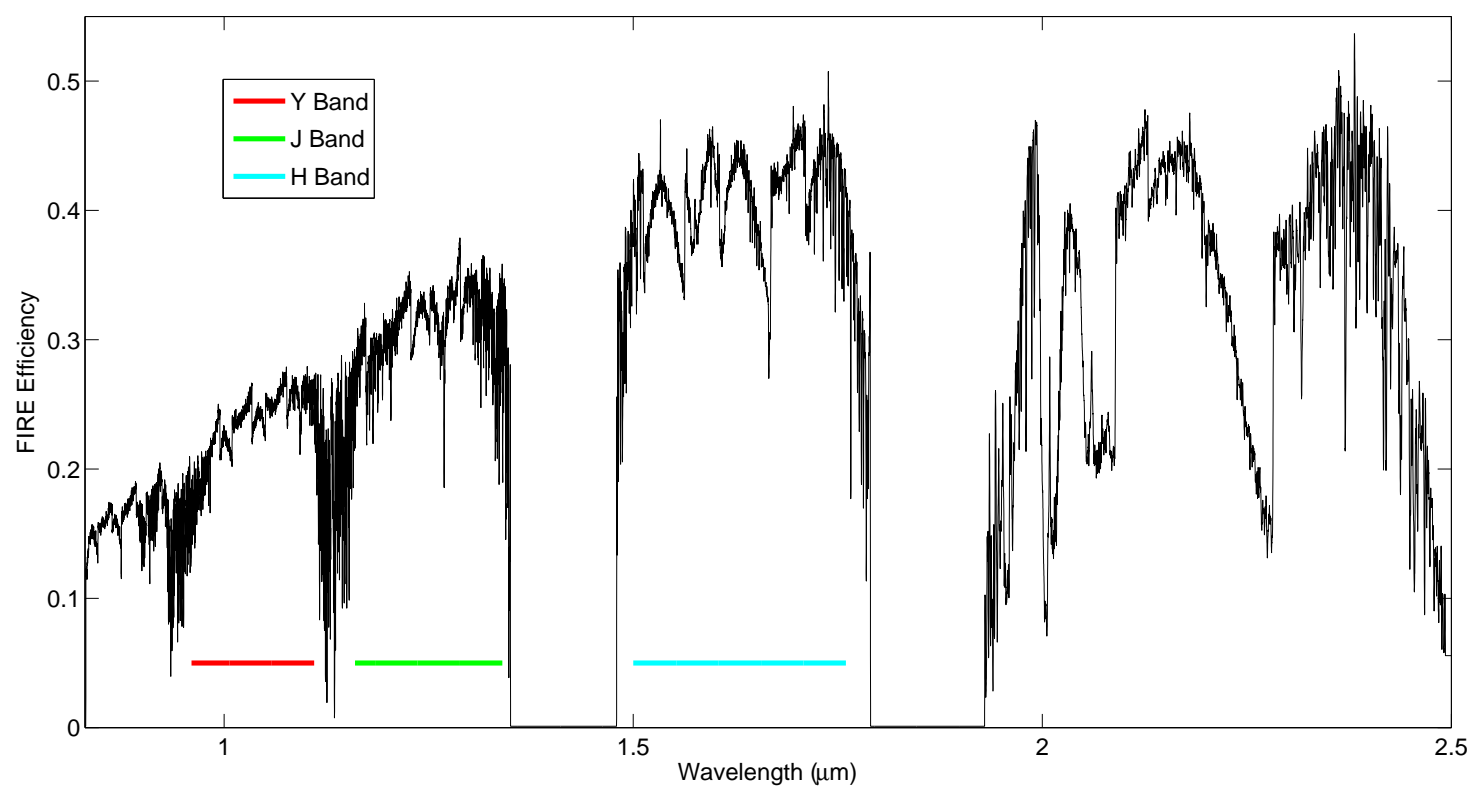

Fig. 3.- End-to-end efficiency of FIRE determined from comparing observations of GD 71 to the CalSTIS archive. Both atmospheric transmission and instrument throughput are included in this determination. The colored bars show the spectral regions over which we measured the continuum sky flux. 


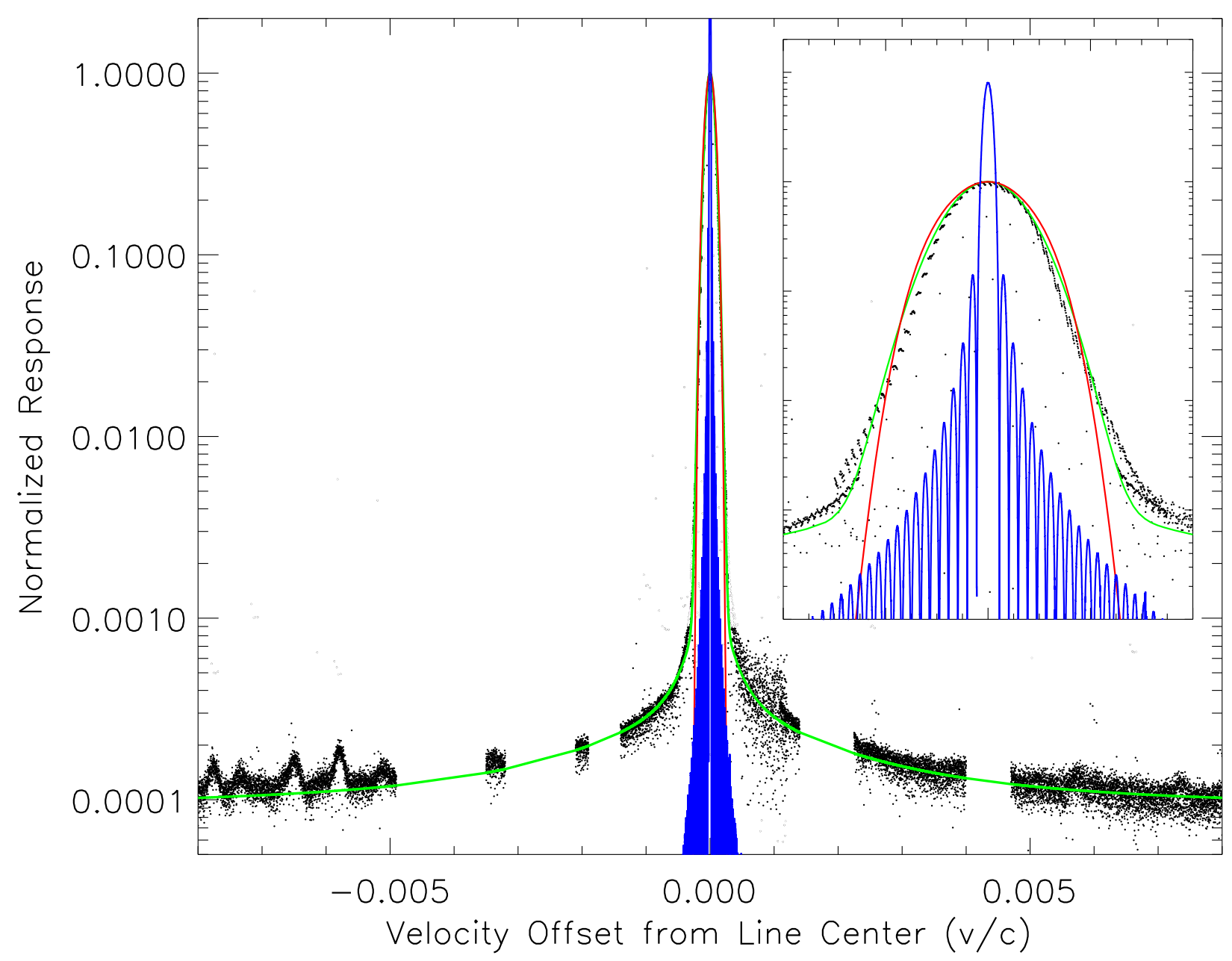

Fig. 4. - The spectral Line Spread Function for FIRE. Measurement from a ThAr lamp is shown by the black points, and a superposition of Gaussian and Lorentzian functions (green) are fitted. The spectral resolution is limited by the entrance slit, so the grating diffraction pattern (blue) does not dominate the LSF. The total flux of the grating pattern is normalized to the area of the Gaussian core (red). 

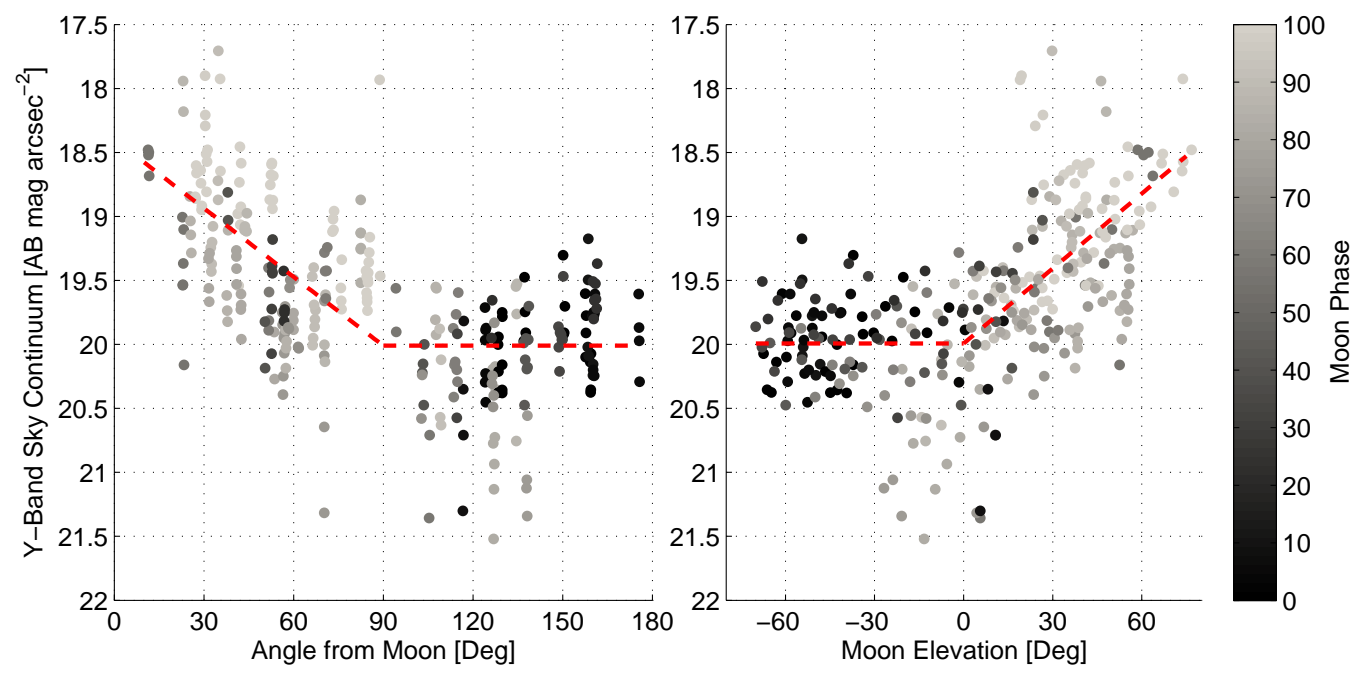

Fig. 5.- In the $Y$ band, angular distance from the moon and moon elevation impact the sky continuum most strongly. The linear least-squares fits were only performed on observations with the moon above the horizon and $>50 \%$ illumination. The mean dark level is plotted for negative moon elevations. The slope of the fits are shown in Table 1 .
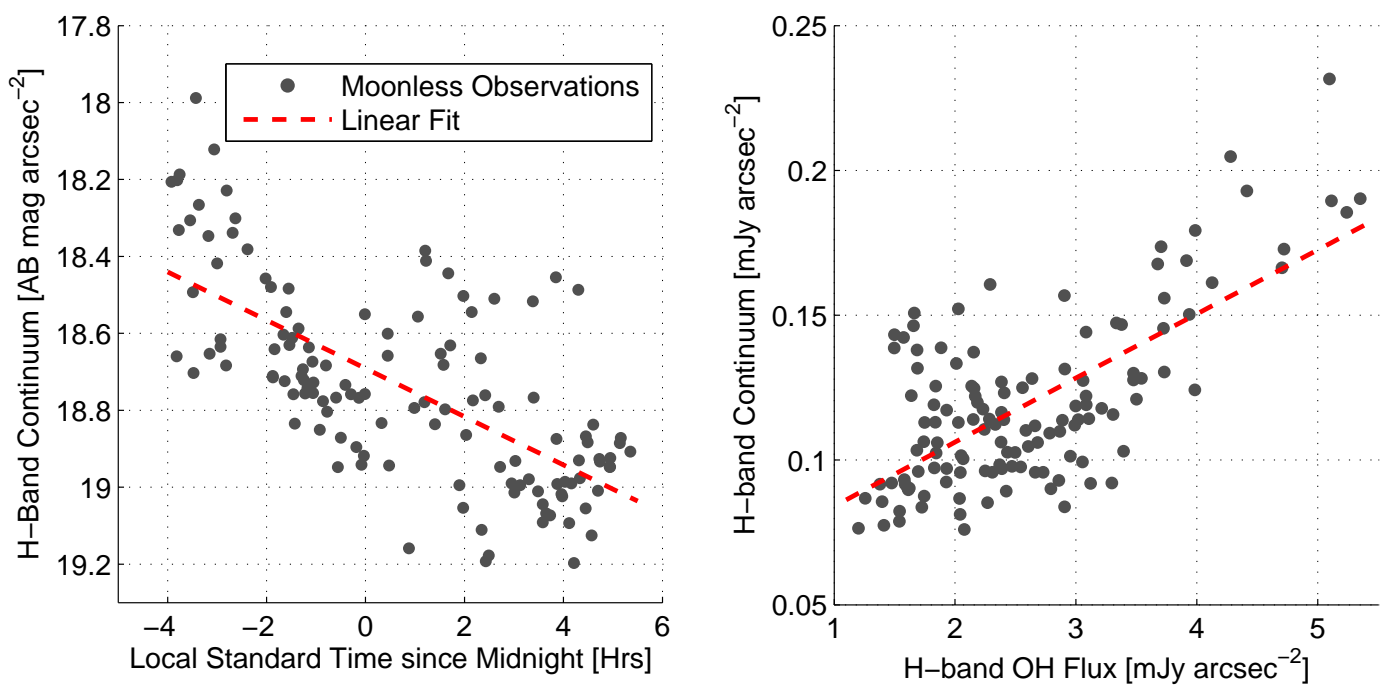

Fig. 6. - In the $H$ band, the local time of night (left) has the largest correlation with continuum flux as the telescope and its surroundings cool down. The $\mathrm{OH}$ flux (right) shows a strong correlation with the continuum measurement as the wings of the LSF allow flux from the $\mathrm{OH}$ lines to scatter into the continuum. The linear least-squares fits were performed only on the moonless observations, and the slopes are shown in Table 1 . 

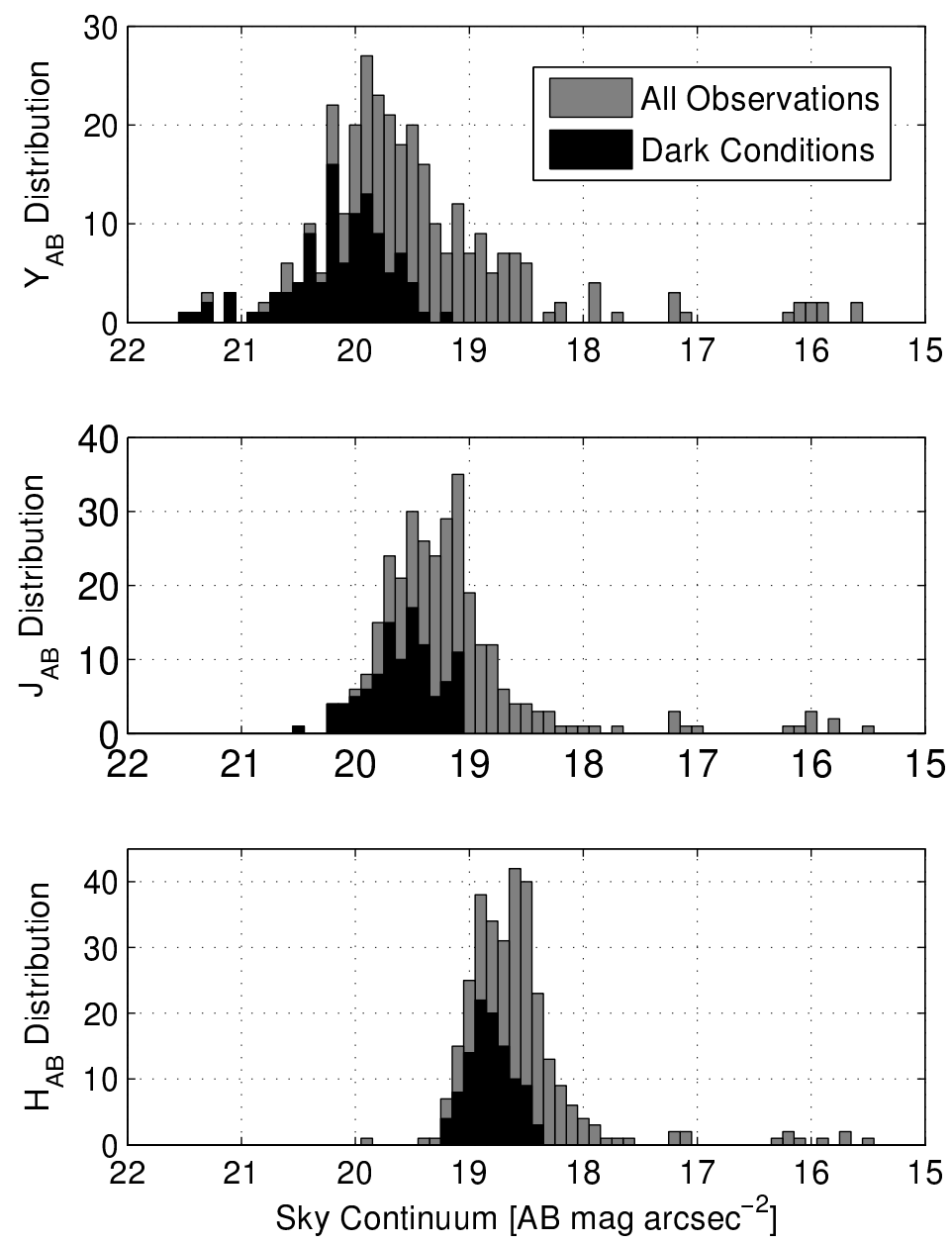

Fig. 7.- Continuum sky background in the $Y, J$, and $H$ bands. The specific spectral regions used for each determination are shown in Figure 3 , 


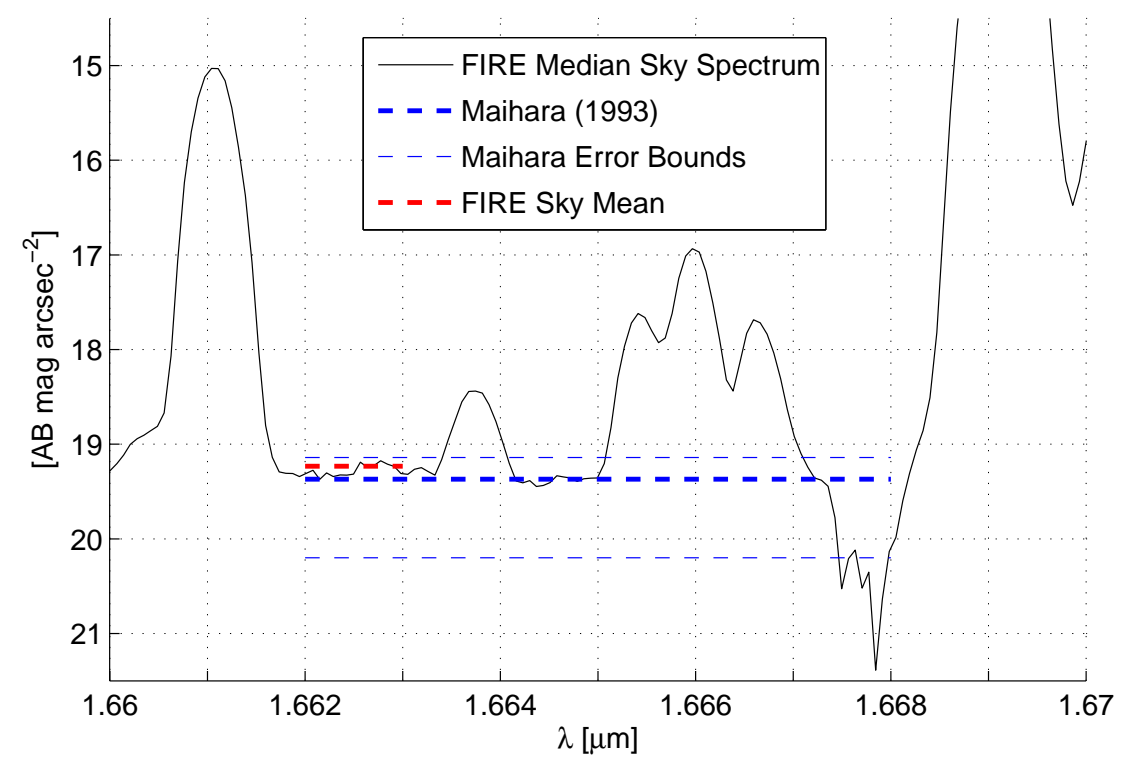

Fig. 8. - The mean sky spectrum over the same region shown in Figure 2 of Maihara et al. (1993). The blue line indicates the sky level measured by Maihara at Mauna Kea, and the red bar indicates the region over which our median sky spectrum is compared to it. The low-level emission and absorption features $( \pm 2 \mathrm{mag})$ are likely due to atmospheric methane. 

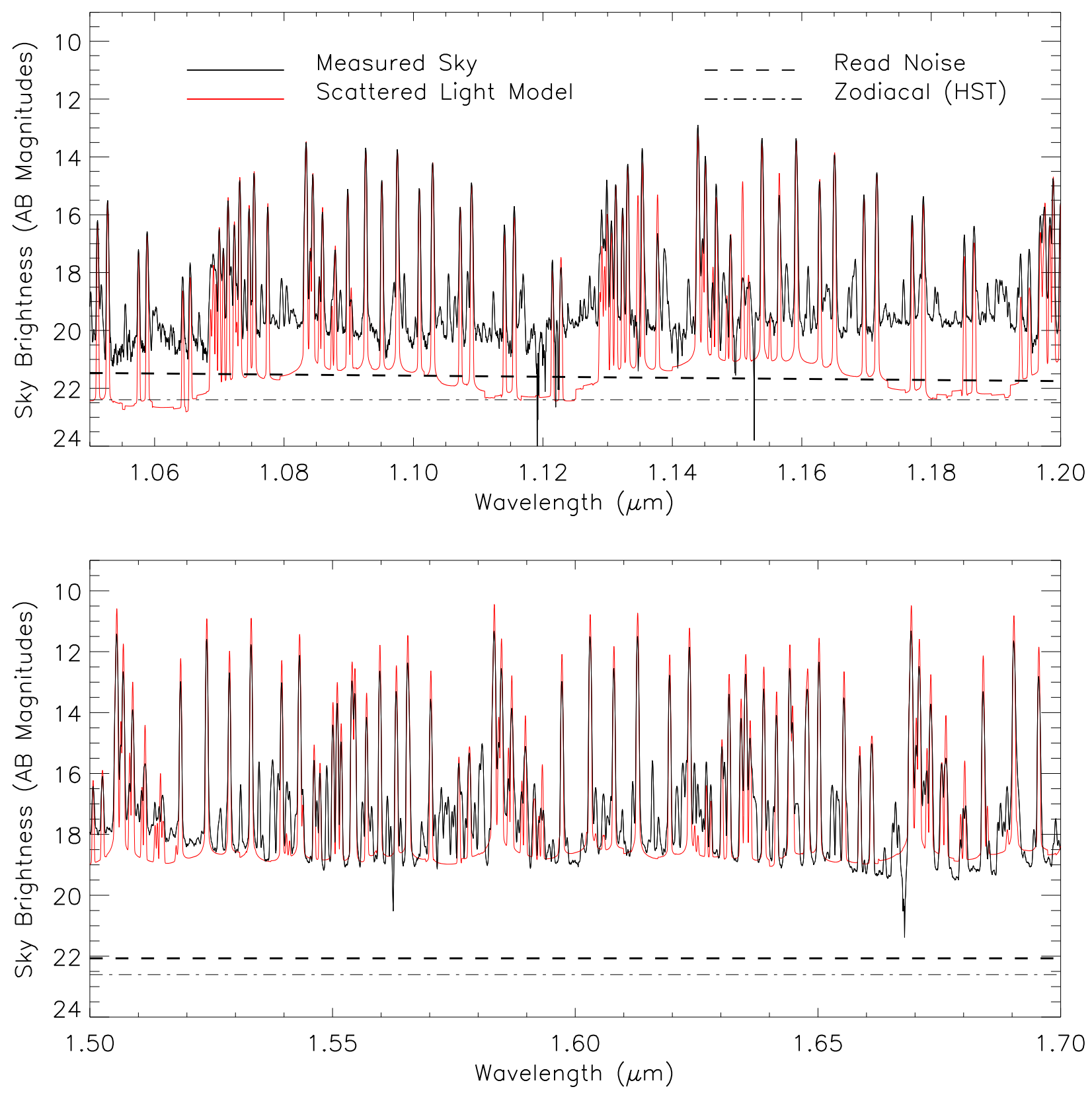

Fig. 9.- In the $H$ band (lower panel), the convolution of our empirically-determined LSF with an $\mathrm{OH}$ linelist (red) shows good agreement with the measured sky spectrum (black). Across the $Y$ and $J$ bands (upper panel), the measured sky spectrum shows a higher continuum level than the LSF convolution would predict. In all three bands, the measured continuum level is well above the read noise floor and Zodiacal light. 


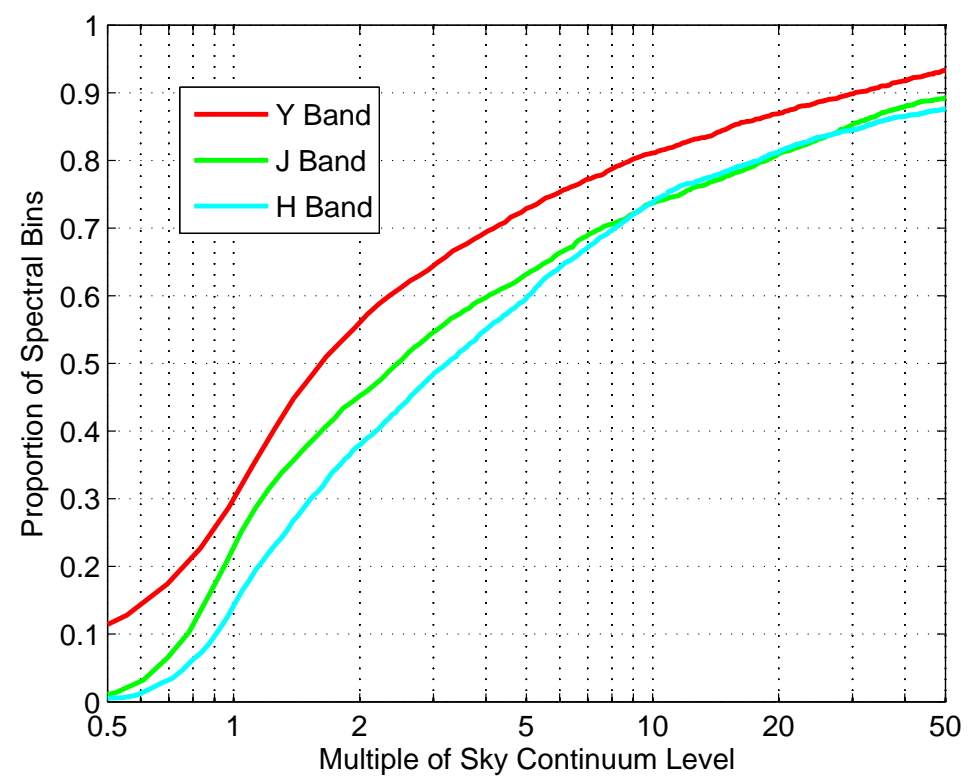

Fig. 10. - The cumulative proportion of spectral bins achieving the sky continuum level, and multiples thereof, are plotted for the $\mathrm{Y}, \mathrm{J}$, and $\mathrm{H}$ bands. The specific spectral regions used for each determination are shown in Figure 3 .

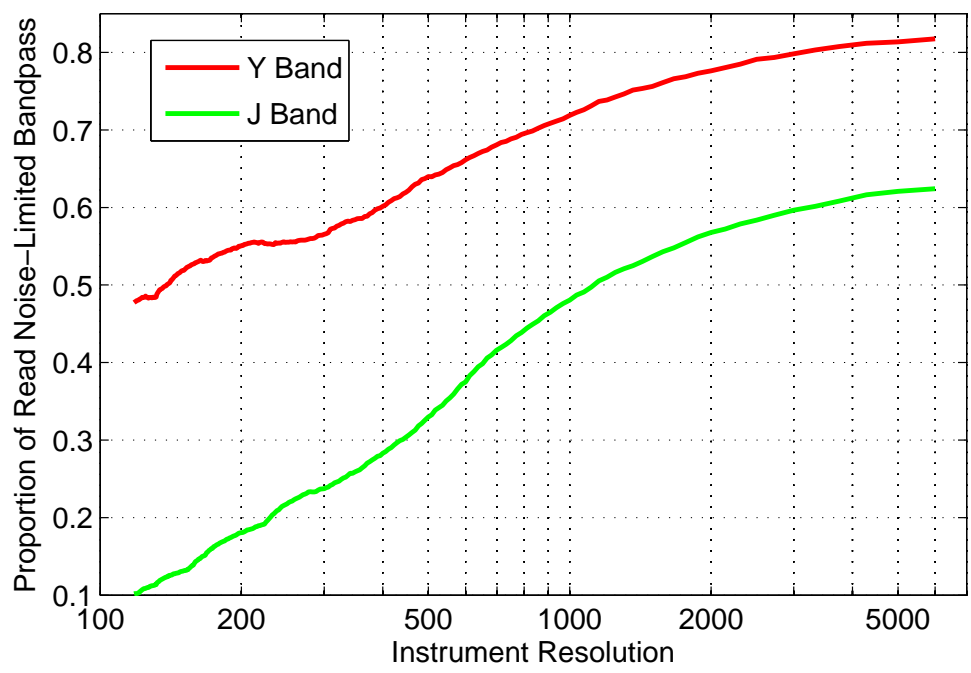

Fig. 11. - The proportion of spectral bins having sky noise lower than the read noise of 7.65 electrons for an exposure of 1500 seconds are plotted against instrument resolution. The data points at $R=6000$ are from FIRE's sky spectrum; all lower resolutions were simulated by convolving the FIRE spectrum with Gaussian kernels of increasing width. 